\title{
Animal models, open science, \& space-omics
}

\author{
Animals are regular inhabitants of the International Space Station, but the knowledge they reveal doesn't just end \\ with their missions. Open science efforts are democratizing space science and helping researchers around the \\ world understand what animal models of spaceflight can reveal about spending time among the stars.
}

\section{Ellen P. Neff}

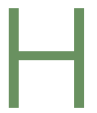
ere on Earth, our hearts are constantly pumping against gravity. Take that force away and our blood no longer wants to pool at our feet; rather, free from Earth's incessant pull, blood pressure shifts a bit towards our heads. Astronauts, as a result, often find themselves with headaches - one of a number of risks inherent to space flight.

Space is an extreme environment. Even in the low Earth orbit that the International Space Station (ISS) occupies, astronauts are subject to more radiation than they experience back on the ground. Microgravity (the Earth still exerts some influence on those living aboard the ISS) meanwhile unloads the mechanical stress on the body, which can cause issues for our musculoskeletal systems - signs of muscle atrophy and bone loss are commonly observed in astronauts after they've returned to earth. Living in extreme confinement with others is no cup of tea for the immune system either, and many astronauts show signs of pre-mature aging after their missions. All of these effects are only likely to be compounded as humans plan for longer and longer missions further and further away from the Earth.

As with studying any human condition, animal models have been valuable in understanding and attempting to mitigate the negative effects of spaceflight. For twenty years now, the ISS has played host not just to astronauts conducting scientific research, but to many animal missions coordinated through space agencies including NASA, the European Space Agency (ESA), the Japan Aerospace Exploration Agency (JAXA), and the Russians State Corporation for Space Activities (ROSCOSMOS).

Space missions are expensive propositions that take years to plan and can yield, relative to experiments conducted back in wet labs on the ground, somewhat smaller sample sizes to analyze across a limited number of time points. To make the most of those precious mice - and flies, worms, even fish, and more - NASA, other international Space Agencies, and those interested in space

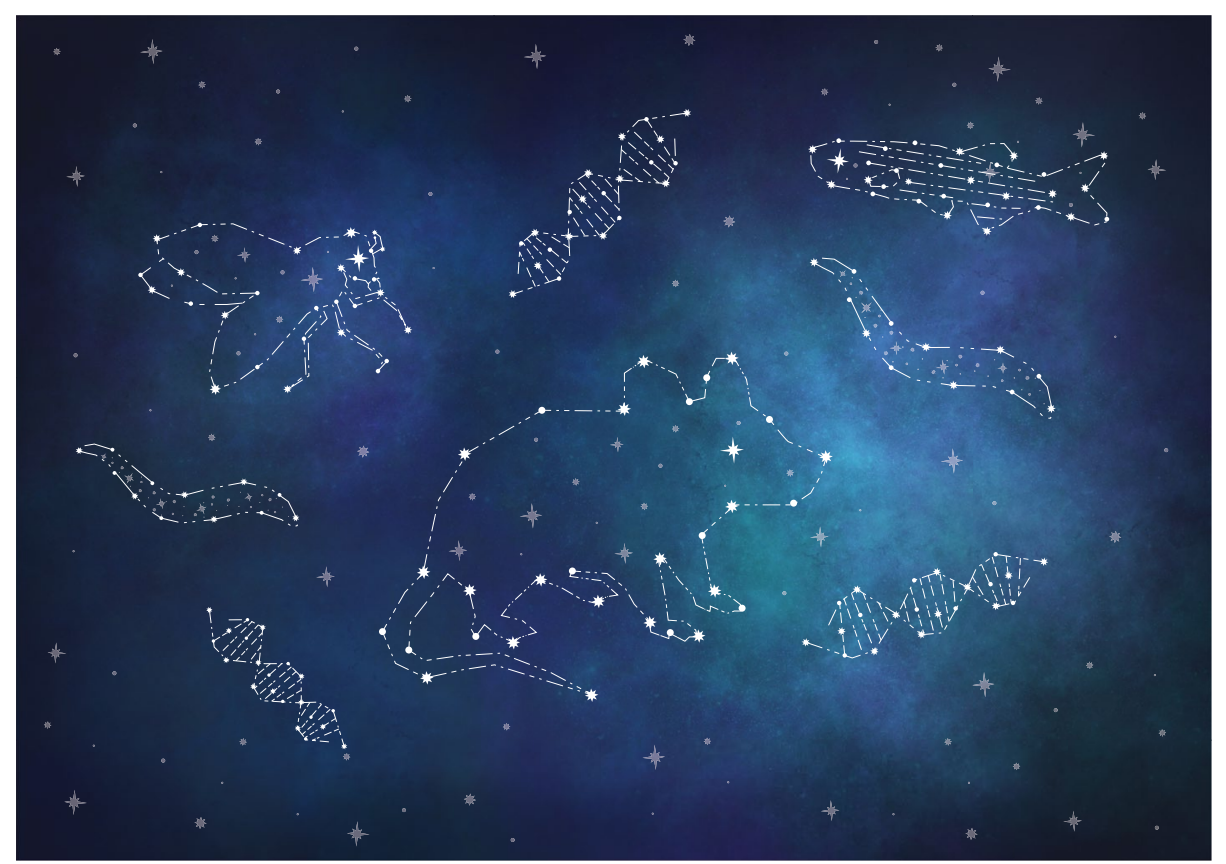

Animal models among the stars. Credit: M. Spence / Springer Nature

biology have been on an open data mission. You don't need to be a bioinformatician - or an astronaut - to get involved.

\section{Space models}

Different animals have long been sent to space, on rockets, during the Space Shuttle Era, and now aboard the ISS to investigate the safety of spaceflight. As on Earth, each comes with different advantages and disadvantages for asking different research questions, but scientists involved with model organism missions over the years have used their space-flown subjects to look at conditions such muscle atrophy and bone loss; changes in the immune system; shifts in the microbiome and how microbes and pathogens interact; sleep disruptions, neuronal changes, aging, and myriad effects on different organs [See Figure 1 in ref. ${ }^{1}$ ].

Studies have involved analyses of changes in different tissues directly, and by analyzing how gene and protein expression, as well as metabolomes and epigenomes, shift in space compared to terrestrial controls. What's being up- or down- regulated in the models could help explain phenotypes and provide a potential target for therapeutic interventions.

Mice, as mammals like us, have been a priority for those who want to understand the effects of spaceflight, and the ISS has regularly welcomed rodent missions. Much care and attention goes into designing rodent habitats for the ISS - these aren't your standard shoeboxes - and for making sure the animals are fed and watered with minimal human intervention.

Other model organisms have also called the ISS home over the years. Zebrafish and medaka can reside in a special Aquatic Habitat Unit built by JAXA, while ESA has sponsored missions with Xenopus frog embryos. Worms and flies meanwhile can fly in simpler set ups and yield larger numbers of subjects than mice or aquatic species. 


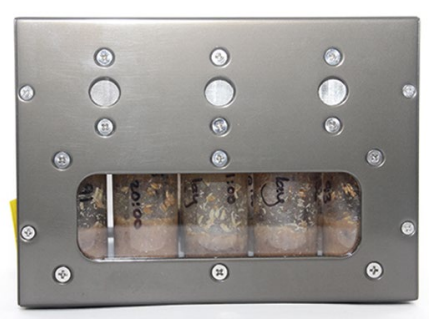

Space-flying flies, ready in their vials. Credit: K. Ocorr / Sanford Burnam Prebys

\section{Drosophila melanogaster can spend} missions within plastic vials along with some food; about 60 flies can inhabit one of these habitats. Karen Ocorr, an assistant professor at Sanford Burnham Prebys who uses flies as a cardiac model, was recently involved with a mission that sent 15 vials within "Vented Fly Boxes," which are about the size of a box of tissues. The vials contained adult flies that reproduced while on orbit; when their middle aged offspring returned a month later, Ocorr and her colleagues saw signs of cardiac dysfunction and remodeling under microgravity along with a number of transcriptomic changes in the fly hearts ${ }^{2}$, which shares similarities with our own.

Caenorhabditis elegans can fly relatively simply as well, and NASA, ESA, and JAXA have all contributed a number of different worm missions over the Nathaniel Szewczyk, a researcher now at the University of Ohio who has worked with teams associated with NASA, JAXA, and ESA over his twenty years of studying C. elegans models of spaceflight, likens the habitats used for several missions as "ketchup packets," filled with culture medium and contained within a box about the size of a deck of cards; these can however yield thousands of worms, reproducing every few days and maturing on orbit.

A more sophisticated setup flew earlier this year as part of the Micro-16 mission. Called NemaFlex, the microfluidics array, developed by Texas Tech engineer Siva Vanapalli, relies on video recordings of the worms as they move through a field of pillars; how much they distend those pillars as they move indicates changes in muscle strength. That mission recently wrapped and when the frozen worms return to Earth, transcriptomic data will be collected for comparison with the behavioral time points - about 300 videos recorded on the ISS, and another 600 from ground controls.

These missions are designed with a particular question in mind and there are numerous logistical challenges to getting animals ready for both the principle investigators (PIs) and space agencies involved - there's hardware \& husbandry to think about, and experimental design considerations that necessitate as little and/or as simple astronaut involvement as possible. The animals then have to make it to the launch site - with COVID-19 delaying deliveries the world over, Vanapalli found himself at the airport explaining why he had packets of worms in his bags (these were backups - the originally shipped worms made it safe, sound, and in time for their February 20th launch from the Wallops Flight Facility in Virginia).

Whenever a mission containing biological samples splashes back down, it's a race against time to retrieve those samples and get them back to the lab before any live animals re-acclimate to the effects of Earths' gravity or frozen samples start to thaw. PIs affiliated with a particular model organism mission will then get to work analyzing the tissues relevant to the question they want to ask - say, a mouse femur, fly heart, or the neurons of worms. What about the rest?

The stories of space models don't end with their missions. Combining data can tell you a whole new story, and efforts are well underway to democratize data access.

\section{Mission: data democratization}

Like many children around the world, Willian da Silveira dreamt of becoming an astronaut. As he grew up, he instead turned to pharmacy, which took him to biophysics and biochemistry and finally on to bioinformatics. While at the Medical University of South Carolina, however, that old dream from his youth in Brazil came knocking. NASA wasn't looking for astronauts, but analysts. The space agency was amassing data from model organisms that had flown to space, and they were inviting omics experts and bioinformaticians to take a look.

Da Silveira got a small grant and some mouse liver transcriptomes. Things immediately looked a bit strange - to him, it as if the mice were diabetic. Da Silveira, now at the Queen's University Belfast, and a growing group of collaborators with diverse areas of expertise from institutions around the US dug into more omics data from more mice. It was almost overwhelming, da Silveira recalls, but one detail kept popping up over and over. "A lot of things we were seeing were related to mitochondrial metabolism," he says.

Mitochondria - the powerhouses of the cells, as the saying goes - provide us and all other eukaryotes with energy; space, it seems, zaps it away by way of mitochondrial dysregulation, signs of which

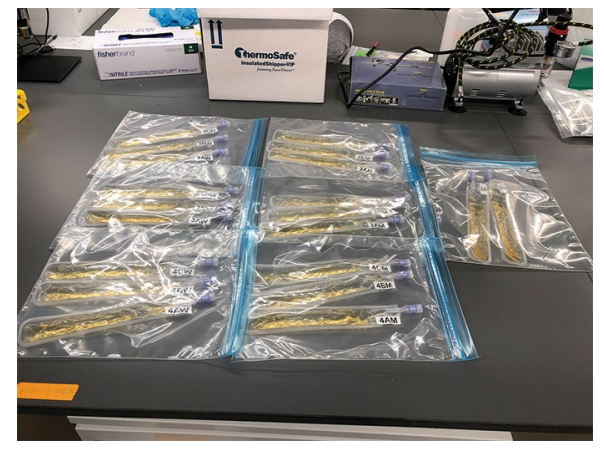

Worm prep: Getting C. elegans ready for spaceflight. Credit: S. Vanapalli / Texas Tech

were evident when the teams analyzed and ran simulations based on the murine gene expression data. From the mice, they looked to the astronauts. Sure enough, when they started looking they found signs that mitochondrial function had gone awry in urine and blood samples from 59 astronauts and in the NASA Twins Study dataset, which compared astronaut Scott Kelly to his Earthbound twin Scott.

The results were published last November in Cell ${ }^{3}$ (alongside 28 other space biology-related papers and commentaries across Cell Press publications) and suggest mitochondrial stress, which can contribute to insulin resistance, premature aging, and immune issues, is a persistent space phenotype that could be a valuable target to mitigate many different ailments that come from living in microgravity and with increased exposure to radiation.

To da Silveira, it was an impossible dream now come true, thanks to an open science project managed through NASA's Ames Research Center in California called GeneLab. "I would not be able to be in the field if not for GeneLab," da Silveira says. "They are making data accessible to anyone in the world."

Since April 2015, NASA has hosted omics datasets and related data from model organism missions in its GeneLab database (non-omics data, meanwhile, has been archived at the Ames Life Sciences Data Archive (ALSDA), which is currently working to improve its integration with $\mathrm{GeneLab}^{4}$ ). These include transcriptomes, proteomes, epigenomes, metagenomes, and metabolomes for model systems including plants, microbes, and animals. The data have been generated and shared by PIs as well produced from in-house analyses of archived tissues from the NASA Space Biology Biospecimen Sharing Program and the NASA Biological Institutional Scientific Collection. As of April 2021, GeneLab contained 316 omics datasets 


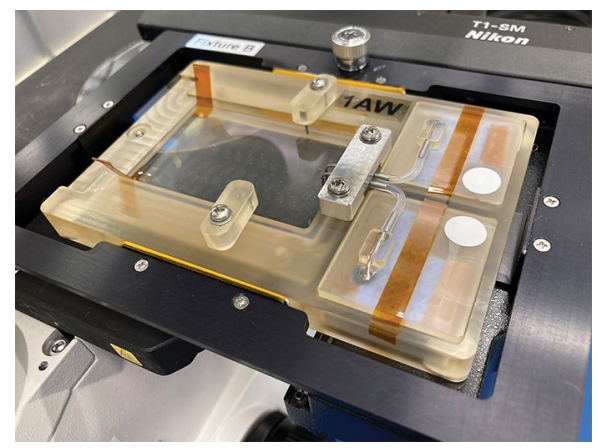

Feel the force: The NemaFlex device. Credit: S. Vanapalli / Texas Tech

that are entirely free for anyone in the world to download. It's data democratization in action, says Project Manager Sylvain Costes.

Both GeneLab and the ALSDA are FAIR (Findable, Accessible, Interoperable, and Reusable) compliant databases designed to be openly available. Such endeavors usually involve a bit of a culture shift, but PIs involved with space missions understand the value "GeneLab has found that their PIs are eager to share their data, because they understand that after their original experiment was conducted, those datasets - because they are spaceflight relevant are absolutely precious," says Ryan Scott, a scientist at Ames working for KBR.

In its early years, those omics data were a bit raw and required some bioinformatics expertise to process and analyze, which can be done in slightly different ways. Standards, however, are taking shape to help anyone - regardless of their background - re-use the omics data captured from space-flown models.

\section{Setting standards}

Standards are crucial for data re-use; without them, it can be hard to make comparisons across different experiments and draw meaningful conclusions about the effects of spaceflight, particularly when the number of subjects involved is relatively small.

"GeneLab is kind of the glue that is actually making Rodent Research a continuum," says Yasaman Shirazi, the ISS Mission Scientist for NASA's Rodent Research, rather than a collection of separate missions of just 20 to 40 animals.

To help set those standards, NASA has convened several working groups, made up of space agency representatives as well as interested researchers with different areas of bioinformatics and biological expertise. "We really want the community working together," says Costes. "We're dealing with such a small number of samples and these experiments are so expensive, we cannot afford not to do it right."

In March, the Analytics Working Group, an interdisciplinary effort to determine how best to analyze and integrate model organism omics data, published their Consensus Pipeline ${ }^{5}$ for RNA-seq experiments; this provides processing details for QC, trimming, mapping, quantification, and differential gene expression (DGE) analysis of transcriptomic data to be uploaded to GeneLab. Interested users can still download raw data, but there are now processed files avaiable and for some datasets, visualizations that more novice users can take advantage of. "People should not be intimidated by these new technologies," says Costes.

Such data standards can indeed make re-use much easier, says Charlotte Nelson, a researcher who worked with GeneLab data as a graduate student in Sergio Baranzini's lab at the University of California San Francisco (UCSF). "I have no background in mouse research," she notes. "Being able to go on to [the GeneLab] website and pull the data - they lay it out in such a way that you understand the different conditions that the mice experience, and in just such a clear way that I haven't seen in many datasets."

She and colleagues at UCSF and NASA Ames recently took rodent research datasets from GeneLab and, after mapping the mouse genes to their human equivalent, ran the expression data through a tool developed for human health bioinformatics called SPOKE. Zooming into the connections between genetic signatures in the mice and human health conditions, the team saw a number of potentially problematic phenotypes, including signs that mice might be experiencing motion sickness and jet $\operatorname{lag}^{6}$ - frequent astronaut ailments. The results, which represent potential genetic targets for countermeasures to treat these issues, are encouraging Nelson and her colleagues to consider analyzing additional animal datasets in SPOKE.

International users are also involved. "We've all learned in the last few years how powerful omics experiments can be in revealing systematic changes at the system level," says Stefania Giacomello, group leader at SciLifeLab in Sweden who is working with different rodent omics datasets from the ISS. Conducting research on orbit comes with unique challenges, but there are a number of points in the space experiment process where protocols could help make sure approaches are syncing up.

Late last year, the International Standards for Space Omics Processing (ISSOP) ${ }^{1}$, a consortium of international researchers and space agency representatives, was established to produce additional standards for how biological data should be generated and preserved in space and then analyzed back on Earth. Members have been meeting regularly, juggling time zones along the way, to identify the problems with conducting biological experiments in space - for example, should a sample be flash frozen or formalin fixed to preserve it? The whole animal, or just a section? What should the habitats look like? The goal is come up with protocols and other standards, including for interoperable metadata needed to average out the noise surrounding a particular experiment, in order to make sure everyone, regardless of where they are from, can be speaking the same space science language. "If we are able to standardize experiments and sample collection, other researchers around the world can access those repositories and be able to conduct new analyses," says Giacomello, who is currently a ISSOP Science co-chair.

All important considerations as humanity looks to longer space flights, including potential missions back to the moon and onward to Mars. "For the first decade or two of spaceflight, all we knew about was bone loss and muscle loss," says Shirazi. "There are a lot of other systems in the body that are affected that we were not aware of until recently." These include effects on the immune and cardiovascular systems, for example. Figuring out what those are and how to treat and/or prevent them will enable safer spaceflight over longer durations, but there are considerations for our health back on Earth too. Reversing bone loss and muscle atrophy in astronauts might also help patients with osteoporosis and different muscular dystrophies, for example; patients on bedrest often develop cardiac myopathies while mitochondrial dysfunction presents in a number of rare diseases.

"The research that is being done on ISS, we call it 'off the Earth, for the Earth," says Shirazi. Model organisms will be right there along the way.

Ellen P. Neff $\bowtie$
Lab Animal.
$\bigotimes_{\text {e-mail: ellen.neff@us.nature.com }}$

Published online: 21 May 2021

https://doi.org/10.1038/s41684-021-00776-1

\footnotetext{
References

1. Rutter, L. et al. Patterns 1, 100148 (2020).

2. Walls, S. et al. Cell Rep 33, 108445 (2020).

3. Da Silveira, W. A. et al. Cell 183, 1185-1201 (2020).

4. Scott, R. T. et al. Cell Rep 33, 108441 (2020)

5. Overbey, E. G. et al. iScience 24, 102361 (2021).

6. Nelson, S. A. et al. Life 11, 42 (2021).
} 